\title{
CCR2 Antagonist CCX872-B
}

National Cancer Institute

\section{Source}

National Cancer Institute. CCR2 Antagonist CCX872-B. NCI Thesaurus. Code C120302.

An orally available human C-C chemokine receptor type 2 (CCR2) antagonist, with potential immunomodulating and antineoplastic activities. Upon oral administration, CCR2 antagonist CCX872-B specifically binds to CCR2 and prevents the binding its cognate endothelium-derived chemokine ligand CCL2 (monocyte chemoattractant protein-1 or MCP1). This may result in the inhibition of both CCR2 activation and CCR2mediated signal transduction, which may inhibit inflammatory processes, angiogenesis, tumor cell migration, and tumor cell proliferation. The G-protein coupled receptor CCR2 is expressed on the surface of monocytes and macrophages, and stimulates their migration and infiltration; it plays a key role in inflammation. CCR2 is overexpressed in certain cancer cell types, where it is involved in angiogenesis, tumor cell migration and proliferation. 